\title{
A Case of Proximal Focal Femoral Deficit Managed with Uniplanar External Fixator Application (Limb Reconstruction System)
}

\author{
Gajanan Pisulkar ${ }^{1}$, Dhruva Utkarsh Angachekar², Bhushan Patil ${ }^{3}$, Mohit Dadlani ${ }^{4}$, Amit Saoji ${ }^{5}$ \\ 1, 2,3,4,5 Department of Orthopaedics, Jawaharlal Nehru Medical College, \\ Datta Meghe Institute of Medical Sciences, Sawangi, Wardha, Maharashtra, India.
}

\section{INTRODUCTION}

Limb elongation surgeries have been described as an important modality for the management of proximal focal femoral deficiencies. Limb reconstruction systems are available to perform these limb lengthening procedures.

Congenital focal femoral deficiency is a clinical condition that was first described by Aitken. They are congenital disfigurements of the lower extremities, mostly involving proximal femur. However, they are clearly distinguishable from isolated coxa vara ${ }^{1}$. It has clinical presentations varying from short femur in mild cases to coxa-vara deformity seen in extreme cases. Other presentations include pseudoarthrosis in the proximal femur, hypoplasia of the lateral femoral condyle, absent cruciate ligaments of knees, muscle hypoplasia, and sponge like network of vessels in the proximal femoral plate. ${ }^{1,2}$

Limb lengthening is performed in patients with Paley's type $1 \mathrm{a}$ and $1 \mathrm{~b}$ congenital femoral deficiency. In cases with Paley Type 2a congenital femoral deficiency, treatment options include knee arthrodesis along with extension prosthesis fitting. Van des rotationplasty, Symes amputation or ablative techniques are the other surgical modalities available.3,4

Current literature does not provide us with just a single treatment of choice which is completely safe and successful for limb lengthening procedure in congenital proximal focal femoral deficiency patients. Limb reconstruction system is an excellent method for femoral lengthening by external fixation technique. ${ }^{5}$ Here we present a case of a female child, aged seven with unilateral proximal femoral focal deficiency managed with LRS technique.

\section{PRESENTATION OF CASE}

A female child, of age seven came to the OPD with complaints of limb length discrepancy and right sided limb shortening since 1 year of age. There was shortening of the right thigh which gradually increased over 6 years. The patient walked with a limp and the opposite side knee and hip flexed.

The patient's mother gave no history of any teratogenic drug use at the time of pregnancy or any perinatal and post-natal complications. The patient was examined and was found to have shortening of $8 \mathrm{~cm}$ in the right limb. The shortening was located in the proximal femoral segment. AP and lateral radiographs were taken (Figure 1). There was shortening of the proximal femur segment with anterolateral bowing of the femur. The femoral head was present and located inside the acetabulum. There was varus deformity at the subtrochanteric level. No pseudoarthrosis was present.
Corresponding Author: Dr. Bhushan Patil. Department of Orthopaedics, Jawaharlal Nehru Medical College, Datta Meghe Institute of Medical Sciences, Sawangi Meghe, Wardha, Maharashtra, India. E-mail: bhushann1001@gmail.com

DOI: $10.14260 /$ jemds/2020/697

How to Cite This Article:

Pisulkar G, Argahekar DU, Pabil B, et al. A case of proximal focal femoral deficit managed with uniplanar external fixator application (limb reconstruction system). J Evolution Med Dent Sci 2020;9(42):31783180, DOI: 10.14260/jemds/2020/697

Submission 15-07-2020,

Peer Review 10-09-2020,

Acceptance 16-09-2020,

Published 19-10-2020.

Copyright (c) 2020 Gajanana Pisulkar et al. This is an open access article distributed under Creative Commons Attribution License [Attribution 4.0 International (CC BY 4.0)] 
The patient was diagnosed with Aitkens type I proximal focal femoral deficiency. The patient was planned for limb lengthening surgery. Unilateral external fixation using LRS after corticotomy for distraction osteogenesis was the surgical plan. The patient was taken up for surgery after full anaesthetic work up. Through an anterolateral incision distal metaphyseal osteotomy of the femur was performed. 3 pins were put in the proximal fragment and 3 pins in the distal fragment (Figure 2). Distraction was started at the rate of the $1 \mathrm{~mm} /$ day by increasing it by $0.25 \mathrm{~mm} 4$ times / day. 7 days later, the distal fragment was going into varus. So, revision was done, and 2 additional pins were put in the distal fragment and distraction was continued at $1 \mathrm{~mm}$ per day. At 21 day follow up distraction was found to be satisfactory, but callus was immature, so distraction was reduced to $0.5 \mathrm{~mm}$ per day (Figure 3). At 2 months follow up distraction was found to be satisfactory with limb lengthening of $6.5 \mathrm{~cm}$. Distraction was stopped at this point and the LRS was removed at 4 months when hard callus was formed. The remaining $1.5 \mathrm{~cm}$ shortening was managed by giving the patient a heel raise. The patient and her family were satisfied with the results.

\section{DISCUSSION}

Paley D., Guardo F. in their chapter of limb lengthening surgery in proximal femoral focal deficiency have described the use of Orthofix LRS for the lengthening of the femur in proximal femoral focal deficiency. They described the surgical protocol for using LRS in lengthening of femur and correction of associated knee values. The LRS bar is kept in line with the femoral shaft and sand witch types clamps are used for distraction. A swivel clamp is used to correct the rotational deformity. It is a good procedure with good results. ${ }^{6}$

Daniel prince et al. in their retrospective study of the utilization of the external fixator in femoral lengthening in proximal femoral focal deficiency found out that uniplanar external fixator like LRS provide excellent results in these patients. 32 patients were part of this study. They underwent distraction osteogenesis of the femur with the help of a uniplanar external fixator. The study included cases which had Paley types 1a, 1b or 2a proximal femoral focal deficiency with stable knee joint as well as hip joint. Patients who have had previous limb lengthening procedures were excluded from the study. 30 ( $94 \%$ ) out of the 32 patients had a follow up of more than 2 years (mean, 3 years; range, $2-4.5$ years). Outcome scores, radiographs of the affected limb, complications and physiotherapy data was analysed both pre and post operatively. The normal limb was taken as a reference while checking for growth inhibition in the affected limb by using serial radiographs over a period of 2 years. Functional and emotional outcomes were analysed utilizing the Paediatric Orthopaedic Society of North America Paediatric Outcomes Data Collection Instruments (PODCI). Post-operative mean femoral lengthening was observed to be of $6 \mathrm{~cm}$, where standard deviation was $\pm 2 \mathrm{~cm}$ and the range lies between $1.6-9 \mathrm{~cm}$ with an average femoral length correction of $112 \%$ (SD $\pm 55 \%$; range, $15 \%-215 \%$ ). The pre and post-operative knee and hip flexion and extension scores were not statistically distinguishable. Patients had favourable standardized scores in accordance to the 6 categories of PODCI. Global functioning and worse pain scores were observed to be lower in patients who underwent femoral lengthening of more than six $\mathrm{cm}$. Similar global functioning scores along with bad pain scores were seen in patients who underwent femoral lengthening more than twenty-five $\%$ of presenting femur length. The study concluded that unilateral external fixator with distraction osteogenesis is a good technique for the management of proximal focal femoral deficiency as has been used in our case. ${ }^{5}$

Adel Mohammed Salama in his study found that femoral elongation procedures in patients with Paley type 1a proximal focal femoral deficiency are fruitful and all around, endured successfully. 16 cases ( 10 males and 6 females) were taken in this study. All cases had Paley's type 1a proximal focal femoral deficiency affecting a single limb. They underwent limb lengthening by distraction osteogenesis using a uniplanar LRS. This prospective study had an average follow-up duration of 36 months. Clinical and functional outcomes of the cases were analysed using The Paediatric Orthopaedic Society of North America PODCI score. Growth suppression or stimulation in the affected limb was observed after removing the LRS and a follow up of 2 years was kept after removal using serial radiographs. Mean femoral lengthening was of $6.5 \mathrm{~cm}$ where SD $\pm 3 \mathrm{~cm}$, with a mean correction in the femoral length discrepancy of $105 \%$ (SD \pm $45 \%)$ at the end of the follow up period. 6 patients (37.5\%) showed over lengthening of the femur by $2-4 \mathrm{~cm} .10$ patients $(62.5 \%)$ had no limb length discrepancy with both femurs of the same length. He concluded that, "femoral elongation in patients with Paley Type 1a proximal femoral deficiency is a successful and well-tolerated procedure". ${ }^{7}$
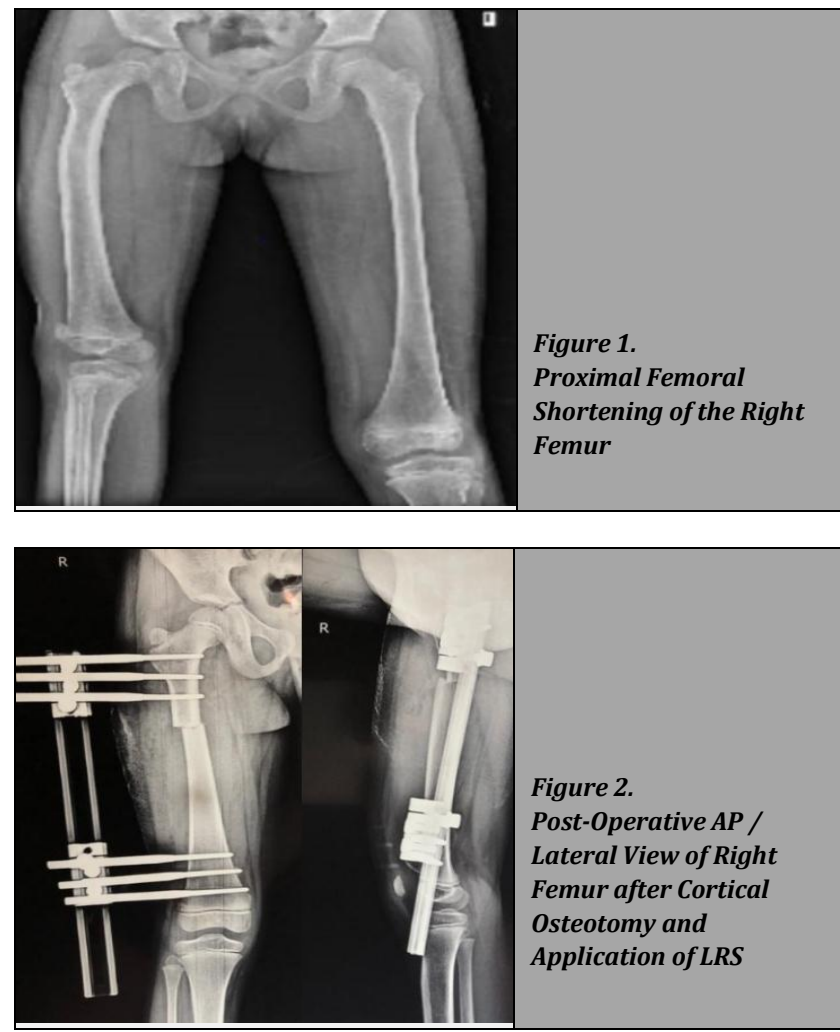

Vivian L. Szymczuk et al conducted a retrospective study to compare lengthening of the femur in proximal focal femoral deficiencies using an external fixator or a magnetic 
intramedullary nail. 62 patients had taken part in this study with 32 being managed with monolateral external fixation application, they were named group A, while group B consisted of 30 patients who were managed with internal lengthening nail. The average age for group A and B was $9.4 \pm$ 3.8 years and $15.4 \pm 4.9$ years, respectively. Mean lengthening achieved in group A and B was $5.6 \pm 1.7$ and $4.8 \pm 1.4 \mathrm{~cm}$, respectively. Mean follow-up for groups A and B was $4.47 \pm$ 2.7 years and $1.86 \pm 0.7$ years, respectively. The average distraction index showed $0.7 \pm 0.2 \mathrm{~mm} / \mathrm{d}$ and $0.7 \pm 0.2 \mathrm{~mm} /$ $\mathrm{d}$ for groups A and B respectively $(\mathrm{p}=0.99)$. Meanwhile, the mean consolidation index for group A showed $29.3 \pm 12.7$ and $34.8 \pm 11.2 \mathrm{~d} / \mathrm{cm}$ for group B accordingly $(\mathrm{p}=0.08)$. No difference was established between the mean arc of motion of the 2 groups. Towards the end of distraction and consolidation the range of motion was found to be lower in group A as compared to group B. Statistically both groups had similar complications. However, pin site infections were a common complication in case of use of external fixator. The study concluded that though intramedullary magnetic nailing is superior to LRS good results are obtained even with the use of uniplanar external fixator. ${ }^{8}$

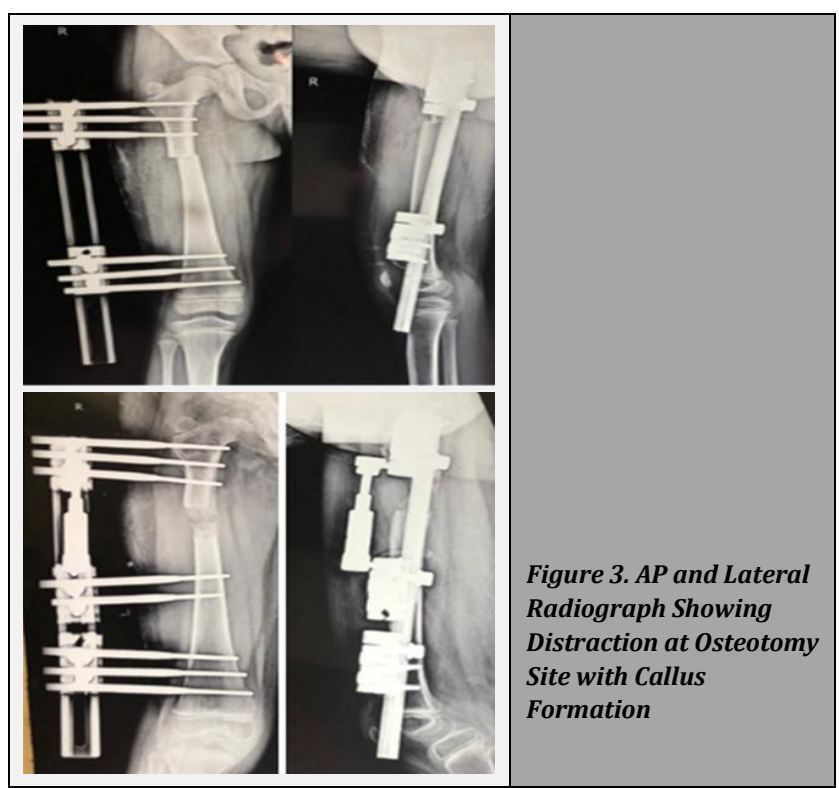

\section{CONCLUSIONS}

Limb reconstruction system is an effective treatment modality in the management of proximal focal femoral deficiencies. It provides excellent clinical results and postoperative patient satisfaction. Hip and knee range of motion is also excellent with this technique.

Financial or other competing interests: None.

Disclosure forms provided by the authors are available with the full text of this article at jemds.com.

\section{REFERENCES}

[1] Aitken GT. Proximal femoral focal deficiency. In: Limb development and deformity: problems of evaluation and rehabilitation.Springfield II: Charles C Thomas Publisher1969:456-76.

[2] Amstutz HC, Wilson PD. Dysgenesis of the proximal femur (coxavara) and its surgical management. JBone Joint Surg Am 1962;44(1):1-24.

[3] Ackman J, Altiok H, Flanagan A, et al. Long-term followup of Van Nesrotationplasty in patients with congenital proximal focal femoral deficiency. Bone Joint J 2013;95(2):192-8.

[4] Westberry DE, Davids JR. Proximal focal femoral deficiency (PFFD): management options and controversies. Hip Int 2009;19Suppl6:S18-25.

[5] Prince DE, Herzenberg JE, Standard SC, et al. Lengthening with external fixation is effective in congenital femoral deficiency. ClinOrthopRelat Res 2015;473(10):3261-71.

[6] Paley D, Guardo F. Lengthening reconstruction surgery for congenital femoral deficiency. In: KocaoğluM, ed.Advanced techniques in limb reconstruction surgery.Berlin, Heidelberg:Springer2015:245-99.

[7] Salama AM. Bone lengthening for management of type 1a proximal femoral deficiency with or without varus deformity.American Research Journal of Orthopedics and Traumatology 2016;1:1-8.

[8] Szymczuk VL, Hammouda AI, Gesheff MG. Lengthening with monolateral external fixation versus magnetically motorized intramedullary nail in congenital femoral deficiency. JPediatrOrthop 2019;39(9):458-65. 